PROCEEDINGS OF THE

AMERICAN MATHEMATICAL SOCIETY

Volume 133, Number 1, Pages 239-244

S 0002-9939(04)07649-X

Article electronically published on August 20, 2004

\title{
ON THE UPPER SEMICONTINUITY OF THE WU METRIC
}

\author{
MAREK JARNICKI AND PETER PFLUG
}

(Communicated by Mei-Chi Shaw)

Abstract. We discuss continuity and upper semicontinuity of the Wu pseudometric.

The $\mathrm{Wu}$ pseudometric was introduced by $\mathrm{H}$. Wu in $\mathrm{Wu} 1993$ (and $\mathrm{Wu}]$ ). Various properties of the $\mathrm{Wu}$ metric may be found for instance in Che-Kim 1996, Che-Kim 1997, Kim 1998], Che-Kim 2003], [Juc 2002]. Nevertheless, it seems that even quite elementary properties of this metric are not completely understood, e.g. its upper semicontinuity.

First, let us formulate the definition of the $\mathrm{Wu}$ pseudometric in an abstract setting. Let $h: \mathbb{C}^{n} \longrightarrow \mathbb{R}_{+}$be a $\mathbb{C}$-seminorm. Put:

$I=I(h):=\left\{X \in \mathbb{C}^{n}: h(X)<1\right\}$ ( $I$ is convex $)$,

$V=V(h):=\left\{X \in \mathbb{C}^{n}: h(X)=0\right\} \subset I\left(V\right.$ is a vector subspace of $\left.\mathbb{C}^{n}\right)$,

$U=U(h):=$ the orthogonal complement of $V$ with respect to the standard Hermitian scalar product $\langle z, w\rangle:=\sum_{j=1}^{n} z_{j} \bar{w}_{j}$ in $\mathbb{C}^{n}$,

$I_{0}:=I \cap U, h_{0}:=\left.h\right|_{U}\left(h_{0}\right.$ is a norm, $\left.I=I_{0}+V\right)$.

For any pseudo-Hermitian scalar product $s: \mathbb{C}^{n} \times \mathbb{C}^{n} \longrightarrow \mathbb{C}$, let

$$
q_{s}(X):=\sqrt{s(X, X)}, X \in \mathbb{C}^{n}, \quad \mathbb{E}(s):=\left\{X \in \mathbb{C}^{n}: q_{s}(X)<1\right\} .
$$

Consider the family $\mathcal{F}$ of all pseudo-Hermitian scalar products $s: \mathbb{C}^{n} \times \mathbb{C}^{n} \longrightarrow \mathbb{C}$ such that $I \subset \mathbb{E}(s)$, equivalently, $q_{s} \leq h$. In particular,

$$
V \subset I=I_{0}+V \subset \mathbb{E}(s)=\mathbb{E}\left(s_{0}\right)+V,
$$

where $s_{0}:=\left.s\right|_{U \times U}$ (note that $\mathbb{E}\left(s_{0}\right)=\mathbb{E}(s) \cap U$ ). Let $\operatorname{Vol}\left(s_{0}\right)$ denote the volume of $\mathbb{E}\left(s_{0}\right)$ with respect to the Lebesgue measure of $U$. Since $I_{0}$ is bounded, there exists an $s \in \mathcal{F}$ with $\operatorname{Vol}\left(s_{0}\right)<+\infty$. Observe that for any basis $e=\left(e_{1}, \ldots, e_{m}\right)$ of $U$ $\left(m:=\operatorname{dim}_{\mathbb{C}} U\right)$ we have

$$
\operatorname{Vol}\left(s_{0}\right)=\frac{C(e)}{\operatorname{det} S},
$$

where $C(e)>0$ is a constant (independent of $s$ ) and $S=S\left(s_{0}\right)$ denotes the matrix representation of $s_{0}$ in the basis $e$, i.e. $S_{j, k}:=s\left(e_{j}, e_{k}\right), j, k=1, \ldots, m$. In particular, if $U=\mathbb{C}^{m} \times\{0\}^{n-m}$ and $e=\left(e_{1}, \ldots, e_{m}\right)$ is the canonical basis, then $C(e)$ is the volume of the open unit Euclidean ball $\mathbb{B}_{m} \subset \mathbb{C}^{m}$. We are interested in

Received by the editors October 3, 2003

2000 Mathematics Subject Classification. Primary 32F45.

Key words and phrases. Wu metric.

Both authors were supported in part by KBN grant no. 5 P03A 03321 and by DFG grant no. $227 / 8-1$. 
finding an $s \in \mathcal{F}$ for which $\operatorname{Vol}\left(s_{0}\right)$ is minimal, equivalently, $\operatorname{det} S\left(s_{0}\right)$ is maximal. Observe that if $s$ has this property (with respect to $h$ ), then for any $\mathbb{C}$-linear isomorphism $L: \mathbb{C}^{n} \longrightarrow \mathbb{C}^{n}$, the scalar product

$$
\mathbb{C}^{n} \times \mathbb{C}^{n} \ni(X, Y) \stackrel{L(s)}{\longrightarrow} s(L(X), L(Y)) \in \mathbb{C}
$$

has the extremal property with respect to $h \circ L$. In particular, this permits us to reduce the situation to the case where $U=\mathbb{C}^{m} \times\{0\}^{n-m}$ and next (by restricting all the above objects to $\mathbb{C}^{m} \simeq \mathbb{C}^{m} \times\{0\}^{n-m}$ ) to assume that $m=n$.

Theorem 1 ([Wu], $\mathrm{Wu} 1993])$. There exists exactly one element $s^{h} \in \mathcal{F}$ such that

$$
\operatorname{Vol}\left(s_{0}^{h}\right)=\min \left\{\operatorname{Vol}\left(s_{0}\right): s \in \mathcal{F}\right\}<+\infty .
$$

Put $\widehat{s}^{h}:=m \cdot s^{h}(m:=\operatorname{dim} U(h)), \mathbb{W} h:=q_{\widehat{s}^{h}}$. Obviously, $\mathbb{W} h \leq \sqrt{m} h$ and $\mathbb{W} h \equiv \sqrt{m} h$ iff $h=q_{s}$ for some pseudo-Hermitian scalar product $s$. For instance, $\mathbb{W}\|\|=\sqrt{n}\|\|$, where \|\| is the Euclidean norm in $\mathbb{C}^{n}$. Moreover, $\mathbb{W}(\mathbb{W} h) \equiv \sqrt{m} \mathbb{W} h$.

Remark 2. Assume that $U(h)=\mathbb{C}^{n}$. Let $L: \mathbb{C}^{n} \longrightarrow \mathbb{C}^{n}$ be a $\mathbb{C}$-linear isomorphism such that $|\operatorname{det} L|=1$ and $h \circ L=h$. Then $\operatorname{Vol}\left(s^{h}\right)=\operatorname{Vol}\left(L\left(s^{h}\right)\right)$ and hence $s^{h}=L\left(s^{h}\right)$, i.e. $s^{h}(X, Y)=s^{h}(L(X), L(Y)), X, Y \in \mathbb{C}^{n}$.

Theorem 3 (|Wu|, Wu 1993]

(b) If $h(X):=\max \left\{h_{1}\left(X_{1}\right), h_{2}\left(X_{2}\right)\right\}, X=\left(X_{1}, X_{2}\right) \in \mathbb{C}^{n_{1}} \times \mathbb{C}^{n_{2}}$, then $\widehat{s}^{h}(X, Y)=\widehat{s}^{h_{1}}\left(X_{1}, Y_{1}\right)+\widehat{s}^{h_{2}}\left(X_{2}, Y_{2}\right), \quad X=\left(X_{1}, X_{2}\right), Y=\left(Y_{1}, Y_{2}\right) \in \mathbb{C}^{n_{1}} \times \mathbb{C}^{n_{2}}$, In particular,

$$
\mathbb{W} h(X)=\left(\left(\mathbb{W} h_{1}\left(X_{1}\right)\right)^{2}+\left(\mathbb{W} h_{2}\left(X_{2}\right)\right)^{2}\right)^{1 / 2}, \quad X=\left(X_{1}, X_{2}\right) .
$$

For a domain $G \subset \mathbb{C}^{n}$, let $\mathcal{M}(G)$ denote the space of all pseudometrics

$$
\eta: G \times \mathbb{C}^{n} \longrightarrow \mathbb{R}_{+}, \quad \eta(a ; t X)=|t| \eta(a ; X), \quad a \in G, X \in \mathbb{C}^{n}, t \in \mathbb{C},
$$

such that

$$
\forall_{a \in G} \exists_{M, r>0}: \eta(z ; X) \leq M\|X\|, \quad z \in \mathbb{B}(a, r) \subset G, X \in \mathbb{C}^{n},
$$

where $\mathbb{B}(a, r)$ is the open Euclidean ball centered at $a$ with radius $r$.

For $\eta \in \mathcal{M}(G)$ we define the Wu pseudometric

$$
(\mathbb{W} \eta)(a ; X):=(\mathbb{W} \widehat{\eta}(a ; \cdot))(X), \quad a \in G, X \in \mathbb{C}^{n},
$$

where

$$
\widehat{\eta}(a ; X):=\sup \{h(X): h \text { is a } \mathbb{C} \text {-seminorm, } h \leq \eta(a ; \cdot)\}, \quad a \in G, X \in \mathbb{C}^{n},
$$

denotes the Buseman pseudometric associated to $\eta$ (cf. [J-P 1993], §4.3). Observe that $\mathbb{W} \eta \in \mathcal{M}(G)$.

Recall that an upper semicontinuous metric $\eta \in \mathcal{M}(G)$ is said to be complete if any $\int \eta$-Cauchy sequence is convergent to a point from $G$, where $\int \eta$ denotes the integrated form of $\eta$ (cf. [J-P 1993], $\S \S 4.3,7.3$ ).

\footnotetext{
${ }^{1}$ See also M. Jarnicki, P. Pflug, Invariant distances and metrics in complex analysis - revisited, to appear.
} 
Proposition 4. (a) If $\eta \in \mathcal{M}(G)$ is a continuous metric, then so is $\mathbb{W} \eta$ (cf. Example [6).

(b) If $\eta \in \mathcal{M}(G)$ is a continuous complete metric, then so is $\mathbb{W} \eta$.

(c) If $\left(\delta_{G}\right)_{G}$ is a holomorphically contractible family of pseudometrics (cf. JJ-P 1993]), then for any biholomorphic mapping $F: G \longrightarrow D\left(G, D \subset \mathbb{C}^{n}\right)$ we have

$$
\left(\mathbb{W} \delta_{D}\right)\left(F(z) ; F^{\prime}(z) X\right)=\left(\mathbb{W} \delta_{G}\right)(z ; X), \quad z \in G, X \in \mathbb{C}^{n} .
$$

(d) If $\left(\delta_{G}\right)_{G}$ is a holomorphically contractible family of pseudometrics, then for any holomorphic mapping $F: G \longrightarrow D\left(G \subset \mathbb{C}^{n_{1}}, D \subset \mathbb{C}^{n_{2}}\right)$ we have

$$
\left(\mathbb{W} \delta_{D}\right)\left(F(z) ; F^{\prime}(z) X\right) \leq \sqrt{n_{2}}\left(\mathbb{W} \delta_{G}\right)(z ; X), \quad z \in G, X \in \mathbb{C}^{n},
$$

but, for example, the family $\left(\mathbb{W} \varkappa_{G}\right)_{G}$ is not holomorphically contractible, where $\varkappa_{G}$ is the Kobayashi-Royden pseudometric of $G$ (cf. Example 5 ).

In the case $\eta=\varkappa_{G}$, the above properties (a)-(d) were formulated (without proof) in $\mathrm{Wu}$, $\mathrm{Wu} 1993$.

Proof. (a) Fix a point $z_{0} \in G \subset \mathbb{C}^{n}$. Let $s^{z}:=s^{\widehat{\eta}(z ; \cdot)}, z \in G$. We are going to show that $s^{z} \underset{z \rightarrow z_{0}}{\longrightarrow} s^{z_{0}}$.

By our assumptions, there exist $r>0, c>0$ such that

$$
\eta(z ; X) \geq c\|X\|, \quad z \in \mathbb{B}\left(z_{0}, r\right) \subset G, X \in \mathbb{C}^{n} .
$$

In particular, the sets

$$
I(z):=\left\{X \in \mathbb{C}^{n}: \widehat{\eta}(z ; X)<1\right\}, \quad z \in \mathbb{B}\left(z_{0}, r\right),
$$

are contained in the ball $\mathbb{B}(0, C)$ with $C:=1 / c$. Moreover,

$$
\left|\widehat{\eta}(z ; X)-\widehat{\eta}\left(z_{0} ; X\right)\right| \leq \varphi(z)\|X\|, \quad X \in \mathbb{C}^{n},
$$

where $\varphi(z) \underset{z \rightarrow z_{0}}{\longrightarrow} 0$. Hence

$$
(1+C \varphi(z))^{-1} I(z) \subset I\left(z_{0}\right) \subset(1+C \varphi(z)) I(z), \quad z \in \mathbb{B}\left(z_{0}, r\right),
$$

and consequently,

$$
\begin{aligned}
& I\left(z_{0}\right) \subset(1+C \varphi(z)) \mathbb{E}\left(s^{z}\right)=\mathbb{E}\left((1+C \varphi(z))^{-2} s^{z}\right), \\
& I(z) \subset(1+C \varphi(z)) \mathbb{E}\left(s^{z_{0}}\right)=\mathbb{E}\left((1+C \varphi(z))^{-2} s^{z_{0}}\right), \quad z \in \mathbb{B}\left(z_{0}, r\right) .
\end{aligned}
$$

Hence

$$
\begin{aligned}
& \operatorname{Vol}\left(s^{z_{0}}\right) \leq \operatorname{Vol}\left((1+C \varphi(z))^{-2} s^{z}\right)=(1+C \varphi(z))^{2 n} \operatorname{Vol}\left(s^{z}\right), \\
& \operatorname{Vol}\left(s^{z}\right) \leq \operatorname{Vol}\left((1+C \varphi(z))^{-2} s^{z_{0}}\right)=(1+C \varphi(z))^{2 n} \operatorname{Vol}\left(s^{z_{0}}\right), \quad z \in \mathbb{B}\left(z_{0}, r\right) .
\end{aligned}
$$

Thus $\operatorname{Vol}\left(s^{z}\right) \underset{z \rightarrow z_{0}}{\longrightarrow} \operatorname{Vol}\left(s^{z_{0}}\right)$.

Take a sequence $z_{\nu} \longrightarrow z_{0}$. Since

$$
\left|s^{z_{\nu}}\left(e_{j}, e_{k}\right)\right| \leq \eta\left(z_{\nu} ; e_{j}\right) \eta\left(z_{\nu} ; e_{k}\right), \quad j, k=1, \ldots, n, \nu \in \mathbb{N},
$$

we may assume that $s^{z_{\nu}} \longrightarrow s^{*}$, where $s^{*}$ is a pseudo-Hermitian scalar product. We already know that $\operatorname{Vol}\left(s^{*}\right)=\operatorname{Vol}\left(s^{z_{0}}\right)$. Moreover, by $\left(^{*}\right), I\left(z_{0}\right) \subset \mathbb{E}\left(s^{*}\right)$. Consequently, the uniqueness of $s^{z_{0}}$ implies that $s^{*}=s^{z_{0}}$. 
(b) Recall that $\int \eta=\int \widehat{\eta}$ - cf. [J-P 1993], Proposition 4.3.5(b). By (a), $\mathbb{W} \eta$ is a continuous metric. In particular, the distance $\int(\mathbb{W} \eta)$ is well defined. By Theorem 3(a) we get

$$
\int \widehat{\eta} \leq \int(\mathbb{W} \eta)
$$

which directly implies the required result.

(c) The result is obvious because for any $z \in G$, the mapping $F^{\prime}(z)$ is a $\mathbb{C}$-linear isomorphism and $\delta_{D}\left(F(z) ; F^{\prime}(z) X\right)=\delta_{G}(z ; X), X \in \mathbb{C}^{n}$.

(d) It is known that the family $\left(\widehat{\delta}_{G}\right)_{G}$ is holomorphically contractible (J-P 1993, Theorem 4.3.10(c)). Hence, using Theorem 3(a), we get

$$
\begin{aligned}
\left(\mathbb{W} \delta_{D}\right)\left(F(z) ; F^{\prime}(z) X\right) & \leq \sqrt{n_{2}} \widehat{\delta}_{D}\left(F(z) ; F^{\prime}(z) X\right) \\
& \leq \sqrt{n_{2}} \widehat{\delta}_{G}(z ; X) \leq \sqrt{n_{2}}\left(\mathbb{W} \delta_{G}\right)(z ; X), \quad z \in G, X \in \mathbb{C}^{n} .
\end{aligned}
$$

Example 5. Let $G:=\left\{\left(z_{1}, z_{2}\right) \in \mathbb{B}_{2}:\left|z_{1}\right|<\varepsilon\right\}, 0<\varepsilon<1 / \sqrt{2}$. Recall that $\varkappa_{\mathbb{B}_{2}}(0 ; X)=\|X\|$ and $\varkappa_{G}(0 ; X)=\max \left\{\|X\|,\left|X_{1}\right| / \varepsilon\right\}, X=\left(X_{1}, X_{2}\right)$. Then $\left(\mathbb{W} \varkappa_{\mathbb{B}_{2}}\right)(0 ;(0,1))=\sqrt{2}>\left(\mathbb{W} \varkappa_{G}\right)(0 ;(0,1))$. In particular, the family $\left(\mathbb{W} \varkappa_{D}\right)_{D}$ is not contractible with respect to inclusions.

We point out that Proposition 4 (a) gives us the continuity of $\mathbb{W} \eta$ only in the case where $\eta$ is a continuous metric. It is natural to conjecture that in the general case, where $\eta$ is only an upper semicontinuous (pseudo)metric, $\mathbb{W} \eta$ remains to be upper semicontinuous. The following Example 6 shows that in general this is not true. In the case where $\eta$ is a continuous pseudometric, we do not know whether $\mathbb{W} \eta$ is upper semicontinuous. Observe that the upper semicontinuity (or at least Borel measurability) of $\mathbb{W} \eta$ appears in a natural way when one defines $\int(\mathbb{W} \eta)$. In the case where $\eta=\varkappa_{G}$, the upper semicontinuity of $\mathbb{W} \varkappa_{G}$ is claimed for instance in Wu 1993] (Theorem 1), Che-Kim 1996] (Proposition 2), [Juc 2002] (Theorem 0), but so far there is no proof.

Example 6. There is an upper semicontinuous metric $\eta$ such that $\mathbb{W} \eta$ is not upper semicontinuous.

Indeed, let $\eta: \mathbb{B}_{2} \times \mathbb{C}^{2} \longrightarrow \mathbb{R}_{+}, \eta(z ; X):=\|X\|$ for $z \neq 0$, and $\eta(0, X):=$ $\max \left\{\|X\|,\left|X_{1}\right| / \varepsilon\right\}, X=\left(X_{1}, X_{2}\right) \in \mathbb{C}^{2}(\varepsilon>0$ small). Then $(\mathbb{W} \eta)(z ; X)=\sqrt{2}\|X\|$ for $z \neq 0$, and $\left\{X \in \mathbb{C}^{2}:(\mathbb{W} \eta)(0 ; X)<1\right\} \backslash \mathbb{B}(0,1 / \sqrt{2}) \neq \varnothing$, so $\mathbb{W} \eta$ is not upper semicontinuous (cf. Example 5).

Example 7. There exists a bounded domain $G \subset \mathbb{C}^{2}$ such that $\mathbb{W} \varkappa_{G}$ is not continuous (see Proposition 2 in [Che-Kim 1996], where such a continuity is claimed).

Indeed, let $D \subset \mathbb{C}^{2}$ be a domain such that (cf. [J-P 1993], Example 3.5.10):

- there exists a dense subset $M \subset \mathbb{C}$ such that $(M \times \mathbb{C}) \cup(\mathbb{C} \times\{0\}) \subset D$,

- $\varkappa_{D}(z ;(0,1))=0, z \in A:=M \times \mathbb{C}$,

- there exists a point $z^{0} \in D \backslash A$ such that $\varkappa_{D}\left(z^{0} ; X\right) \geq c\|X\|, X \in \mathbb{C}^{2}$, where $c>0$ is a constant.

For $R>0$ let $D_{R}:=\left\{z=\left(z_{1}, z_{2}\right) \in D:\left|z_{j}-z_{j}^{0}\right|<R, j=1,2\right\}$. It is known that $\varkappa_{D_{R}} \searrow \varkappa_{D}$ when $R \nearrow+\infty$. Observe that $z^{0} \in D_{R}$ and

$$
\varkappa_{D_{R}}\left(z^{0} ; X\right) \geq \varkappa_{D}\left(z^{0} ; X\right) \geq c\|X\|, \quad X \in \mathbb{C}^{2} .
$$


Hence, by Theorem $\left[3(\mathrm{a}),\left(\mathbb{W} \varkappa_{D_{R}}\right)\left(z^{0} ; X\right) \geq c\|X\|, X \in \mathbb{C}^{2}\right.$. In particular,

$$
\left(\mathbb{W} \varkappa_{D_{R}}\right)\left(z^{0} ;(0,1)\right) \geq c .
$$

Fix a sequence $M \ni z_{k} \longrightarrow z_{1}^{0}$. Note that $\left\{z_{k}\right\} \times\left(z_{2}^{0}+R E\right) \subset D_{R}$, which implies that $\varkappa_{D_{R}}\left(\left(z_{k}, z_{2}^{0}\right) ;(0,1)\right) \leq 1 / R, k=1,2, \ldots$ In particular,

$$
\left(\mathbb{W} \varkappa_{D_{R}}\right)\left(\left(z_{k}, z_{2}^{0}\right) ;(0,1)\right) \leq \sqrt{2} \varkappa_{D_{R}}\left(\left(z_{k}, z_{2}^{0}\right) ;(0,1)\right) \leq \sqrt{2} / R, \quad k=1,2, \ldots
$$

Now it clear that if $R>\frac{\sqrt{2}}{c}$, then

$$
\limsup _{k \rightarrow+\infty}\left(\mathbb{W} \varkappa_{D_{R}}\right)\left(\left(z_{k}, z_{2}^{0}\right) ;(0,1)\right) \leq \sqrt{2} / R<c \leq\left(\mathbb{W} \varkappa_{D_{R}}\right)\left(z^{0} ;(0,1)\right),
$$

which shows that for $G:=D_{R}$ the pseudometric $\mathbb{W} \varkappa_{G}$ is not continuous.

Remark 8. We point out the influence of the factor $\sqrt{m}$ in the definition of $\mathbb{W}$ to its upper semicontinuity.

Suppose we defined $\widetilde{\mathbb{W}} h:=q_{s^{h}}, \widetilde{\mathbb{W}} \eta(a ; X)=(\widetilde{\mathbb{W}} \widehat{\eta}(a ; \cdot))(X)$. Then, using the product formula (Theorem $3(\mathrm{~b})$ ), we would get a domain $G \subset \mathbb{C}^{3}$ such that $\widetilde{\mathbb{W}} \varkappa_{G}$ is not upper semicontinuous.

Indeed (the example is due to W. Jarnicki), let $D \subset \mathbb{C}^{2}$ and $D \ni z_{k} \longrightarrow z_{0} \in D$ be such that:

- $\varkappa_{D}\left(z_{k} ; \cdot\right)$ is not a metric (in particular, $\left.m(k):=\operatorname{dim} U\left(\widehat{\varkappa}_{D}\left(z_{k} ; \cdot\right)\right) \leq 1, k \in \mathbb{N}\right)$, - $\varkappa_{D}\left(z_{0} ; \cdot\right)$ is a metric.

Take, for instance, the domain $D$ as in Example 7.

Put $G:=D \times E \subset \mathbb{C}^{3}$. Then $\widetilde{\mathbb{W}} \varkappa_{G}$ is not upper semicontinuous at $\left(\left(z_{0}, 0\right),(0,1)\right)$ because

$$
\begin{aligned}
& \left(\widetilde{\mathbb{W}} \varkappa_{G}\right)^{2}\left(\left(z_{k}, 0\right) ;(0,1)\right)=s^{\varkappa_{G}\left(\left(z_{k}, 0\right) ; \cdot\right)}((0,1),(0,1))=\frac{1}{m\left(z_{k}\right)+1} \geq \frac{1}{2}, \quad k \in \mathbb{N}, \\
& \left(\widetilde{\mathbb{W}} \varkappa_{G}\right)^{2}\left(\left(z_{0}, 0\right) ;(0,1)\right)=s^{\varkappa_{G}\left(\left(z_{0}, 0\right) ; \cdot\right)}((0,1),(0,1))=\frac{1}{m\left(z_{0}\right)+1}=\frac{1}{3} .
\end{aligned}
$$

We conclude the paper by repeating the main open question.

Problem. Let $\eta \in\left\{\gamma_{G}^{(k)}, A_{G}, \varkappa_{G}\right\}$ (cf. [J-P 1993]). Is $\mathbb{W} \eta$ upper semicontinuous?

\section{REFERENCES}

[Che-Kim 1996] C. K. Cheung, K. T. Kim, Analysis of the Wu metric. I: The case of convex Thullen domains, Trans. Amer. Math. Soc. 348 (1996), 1429-1457. MR1357392 (96i:32026)

[Che-Kim 1997] C. K. Cheung, K. T. Kim, Analysis of the Wu metric. II: The case of non-convex Thullen domains, Proc. Amer. Math. Soc. 125 (1997), 1131-1142. MR1363414 (97f:32029)

[Che-Kim 2003] C. K. Cheung, K. T. Kim, The constant curvature property of the Wu invariant metric, Pac. J. Math. 211 (2003), 61-68. MR2016590

[J-P 1993] M. Jarnicki, P. Pflug, Invariant Distances and Metrics in Complex Analysis, de Gruyter Expositions in Mathematics 9, Walter de Gruyter 1993. MR1242120 (94k:32039)

[Juc 2002] P. Jucha, The Wu metric in elementary Reinhardt domains, Univ. Iag. Acta Math. 40 (2002), 83-89. MR1962715 (2003m:32008)

[Kim 1998] K. T. Kim, The Wu metric and minimum ellipsoids, Proc. 3rd Pacific Rim Geometry Conf. (J. Choe ed.), Monogr. Geom. Topology 25, International Press, Cambridge, 1998, 121-138. MR1751069 (2001f:32017) 
[Wu] H. Wu, Unpublished notes.

[Wu 1993] H. Wu, Old and new invariant metrics on complex manifolds, Several complex variables: Proc. Mittag-Leffler Inst. 1987-88 (J.E. Fornæss ed.), Math. Notes, Princeton Univ. Press 38 (1993), 640-682. MR.1207887 (94a:32038)

Institute of Mathematics, Jagiellonian University, Reymonta 4, 30-059 Kraków, POLAND

E-mail address: jarnicki@im.uj.edu.pl

Institut für Mathematik, Carl von Ossietzky Universität Oldenburg, Postfach 2503, D-26111 Oldenburg, Germany

E-mail address: pflug@mathematik.uni-oldenburg.de 